\title{
Does Retinotopy Influence Cortical Folding in Primate Visual Cortex?
}

\author{
Reza Rajimehr ${ }^{1,2}$ and Roger B. H. Tootell ${ }^{1}$ \\ ${ }^{1}$ NMR Martinos Center, Massachusetts General Hospital, Harvard Medical School, Charlestown, Massachusetts 02129, and ${ }^{2}$ McGovern Institute for Brain \\ Research, Massachusetts Institute of Technology, Cambridge, Massachusetts 02139
}

In humans and other Old World primates, much of visual cortex comprises a set of retinotopic maps, embedded in a cortical sheet with well known, identifiable folding patterns. However, the relationship between these two prominent cortical variables has not been comprehensively studied. Here, we quantitatively tested this relationship using functional and structural magnetic resonance imaging in monkeys and humans. We found that the vertical meridian of the visual field tends to be represented on gyri (convex folds), whereas the horizontal meridian is preferentially represented in sulci (concave folds), throughout visual cortex in both primate species. This relationship suggests that the retinotopic maps may constrain the pattern of cortical folding during development.

\section{Introduction}

During evolution, the primate cerebral cortex has expanded disproportionately, relative to overall brain size. Consequently, the cortical sheet becomes folded, to fit in the limited volume provided by the skull (Welker, 1990). Inward-bending (concave) folds are called "sulci," and outward-bending (convex) folds are called "gyri." In a given species, the exact geometry of these folding patterns is not random (Sereno and Tootell, 2005; Van Essen, 2007); indeed, it is consistent enough so that the major sulci and gyri are named individually (e.g., the calcarine sulcus, the superior temporal sulcus, the fusiform gyrus, etc.).

What determines these consistent folding patterns? Some developmental theories suggest that cortical folding is dictated by the differential growth of cortical layers (Richman et al., 1975) or patterns of subcortical neurogenesis (Kriegstein et al., 2006). An alternative theory has been proposed by Van Essen (1997): the mechanical tension along corticocortical connections is the primary driving force for cortical folding. The tension-based morphogenesis tends to reduce the aggregate length of axonal connections, thereby contributing to the compact wiring of neural circuitry in the brain.

In parallel, several groups have pointed out that the average length of cortical connections is also reduced by mapping adjacent retinotopic values along a common eccentricity value (Chklovskii and Koulakov, 2004; Rajimehr and Tootell, 2007); presumably this mechanism underlies the common "mirror symmetry" in retinotopic areas V1, V2, V3, etc., and the "foveal

Received April 16, 2009; revised June 13, 2009; accepted July 24, 2009.

This research was supported by the National Institutes of Health (Grants R01 MH67529 and R01 EY017081 to R.B.H.T.), the Martinos Center for Biomedical Imaging, the National Center for Research Resources, and the MIND Institute. We thank John Maunsell and two anonymous reviewers for helpful comments on this manuscript.

This article is freely available online through the J Neurosci Open Choice option.

Correspondence should be addressed to Reza Rajimehr, NMR Martinos Center, Massachusetts General Hospital, Harvard Medical School, 149 Thirteenth Street, Room 2301, Charlestown, MA 02129. E-mail: reza@nmr. mgh.harvard.edu.

DOI:10.1523/JNEUROSCI.1835-09.2009

Copyright $\odot 2009$ Society for Neuroscience ～0270-6474/09/2911149-04\$15.00/0 patches" (visual field map clusters) across visual cortex (Wandell et al., 2007).

Collectively, these ideas suggest that the retinotopic organization may constraint the pattern of cortical folding in parts of visual cortex. Specifically, this hypothesis predicts that the border between adjacent, mirror-symmetric retinotopic areas (typically, the representation of the vertical meridian) would become a gyrus, and remaining regions of the retinotopic map (often including the horizontal meridian representation) would become sulci.

This hypothesis arises quite naturally from well known details of the cortical architecture. In visual cortex, the retinotopic representation of the vertical meridian is unique because the vertical meridian forms the "seam" between left versus right visual hemifields, which project to the right versus left hemispheres of the brain, respectively. Thus, the vertical meridian representation forms a single line across the cortical surface, dividing two adjacent, mirror-symmetric maps of the visual field. Corresponding retinotopic loci in each of these paired maps are strongly interconnected with each other, through short-range axons. However, loci along the vertical meridian are singularity points on the map of mirror-symmetric areas, so they lack these short-range, paired connections (connections from the vertical meridian are instead made via long-range callosal axons to the opposed hemisphere). During the development of cortical folds, relatively higher (and perhaps earlier) tension along these short-range axons would pull the paired retinotopic sites toward one another, resulting in a gyrus along the vertical meridian representation (which exercises less "pull" on its own). By this idea, the horizontal meridian would tend to be driven into sulci as a secondary effect, because the horizontal meridian is furthest away from gyri defined by the vertical meridian. Additional effects may also contribute (see Discussion).

Qualitative support for this idea can be quite striking [see also the study by Van Essen (1997)]. For instance in the macaque, the border between the most highly retinotopic areas (V1 and V2) is a vertical meridian representation that runs 
along the crown of two different gyri (Fig. 1). Within the same areas, and the same range of visual field eccentricities, the horizontal meridian representations lie in and near two different sulci (Fig. 1). A similar arrangement is found in V1 and V2 of human visual cortex (Fig. 1).

\section{Materials and Methods}

To test this idea quantitatively throughout retinotopically organized visual cortex, we used functional magnetic resonance imaging (fMRI) to map the retinotopy in two awake macaque monkeys and eight human subjects. These data were analyzed in a cortical surface format, to assess the location of retinotopic maps on gyri and sulci, throughout cerebral cortex. The stimuli were real-life images that were confined to retinotopically specific apertures, presented during fMRI in a blocked design (Rajimehr et al., 2009). The retinotopic stimuli included: horizontal meridian (HM) wedges $\left(10^{\circ}\right.$ radius; $30^{\circ}$ angle), vertical meridian $(\mathrm{VM})$ wedges $\left(10^{\circ}\right.$ radius; $60^{\circ}$ angle $)$, foveal (Fov) disks ( $3^{\circ}$ diameter), and peripheral (Per) rings $\left(10^{\circ}\right.$ inner diameter and $20^{\circ}$ outer diameter).

fMRI data were collected using whole-brain echo-planar imaging $(1.25 \mathrm{~mm}$ isotropic voxels in monkeys; $3.0 \mathrm{~mm}$ isotropic voxels in humans) in a $3 \mathrm{~T}$ horizontal scanner. The monkey scans included an exogenous contrast agent (MION) to increase the contrast/noise ratio (Vanduffel et al., 2001). Throughout the functional scans, all subjects continuously fixated the center of stimulus screen, and eye position was monitored in monkeys using an infrared pupil tracking system (ISCAN). High-resolution anatomical volumes were also collected from the same subjects. We used FreeSurfer (http:// surfer.nmr.mgh.harvard.edu/) to reconstruct the cortical surfaces and to analyze the functional data. Additional details of the experimental procedures and data analysis are described previously (Rajimehr et al., 2009).

\section{Results}

Figure 2 shows the results of meridian mapping in monkeys and humans. The comparison between horizontal versus vertical meridian stimuli shows alternating representations of horizontal and vertical meridians in visual cortex (Fig. 2, right panels). For each vertex of the cortical surface mesh, the curvature value (gyrus-sulcus index) and the logarithmic $\mathrm{P}$ value for "HM versus VM" contrast were extracted, and then these values were plotted for all vertices of the entire cortex (Fig. 2, left panels). The curvature value was defined geometrically by averaging two principal curvatures at each vertex. Consistent with our hypothesis, the vertical meridian representations were more often located on gyri, whereas the horizontal meridian representations

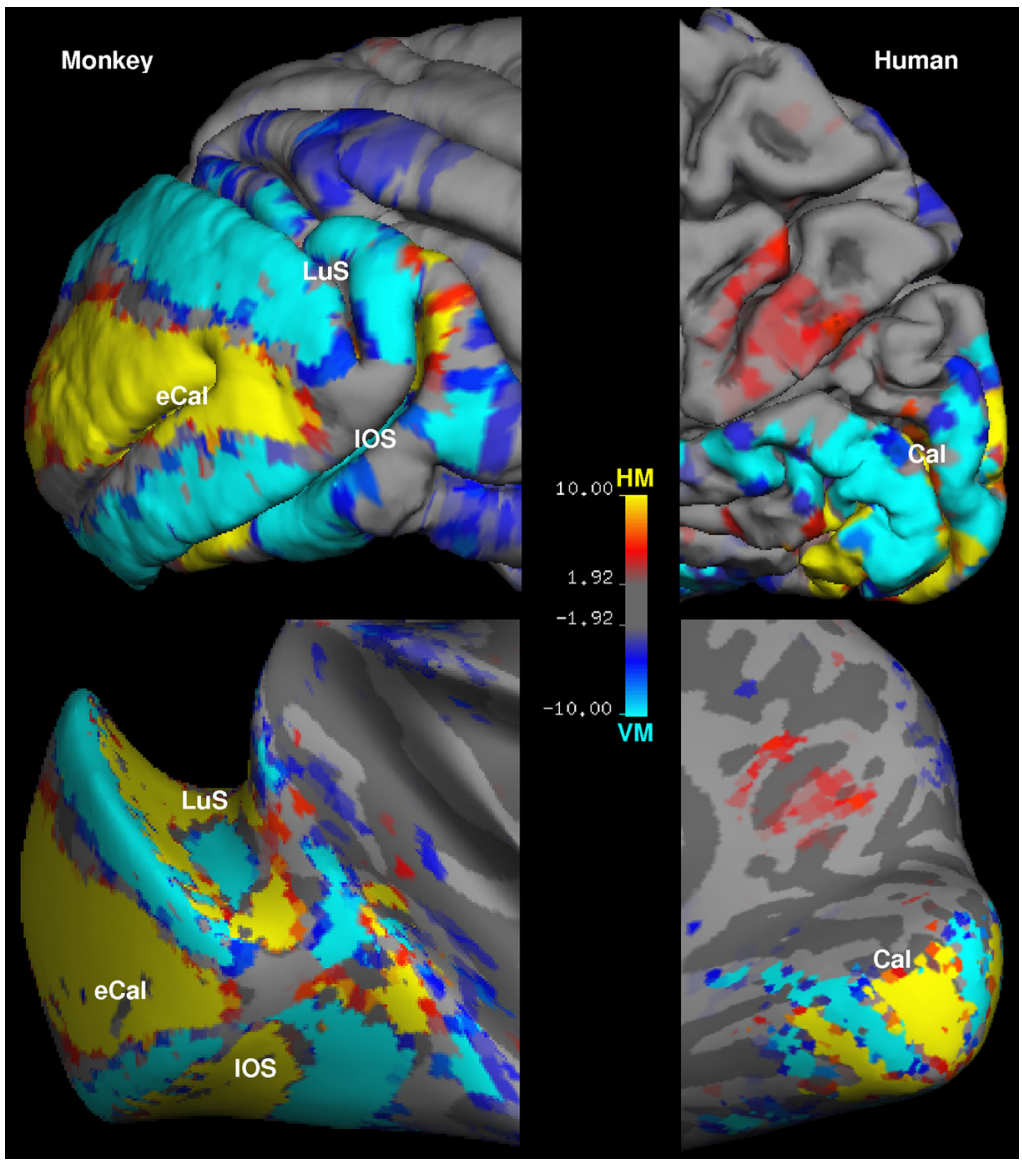

Figure 1. Representation of visual field meridians on folded and inflated cortex. The maps show the relative $\mathrm{fMRl}$ activation for horizontal versus vertical meridian. The activation maps are displayed on a lateral view of folded (top) and inflated (bottom) visual cortex in a macaque monkey (left), and on a medial view of folded (top) and inflated (bottom) visual cortex in a human subject (right). The sulci are indicated as follows: Cal, calcarine; eCal, external calcarine; LuS, lunate; IOS, inferior occipital. The color scale bar indicates the $P$ value in logarithmic format.

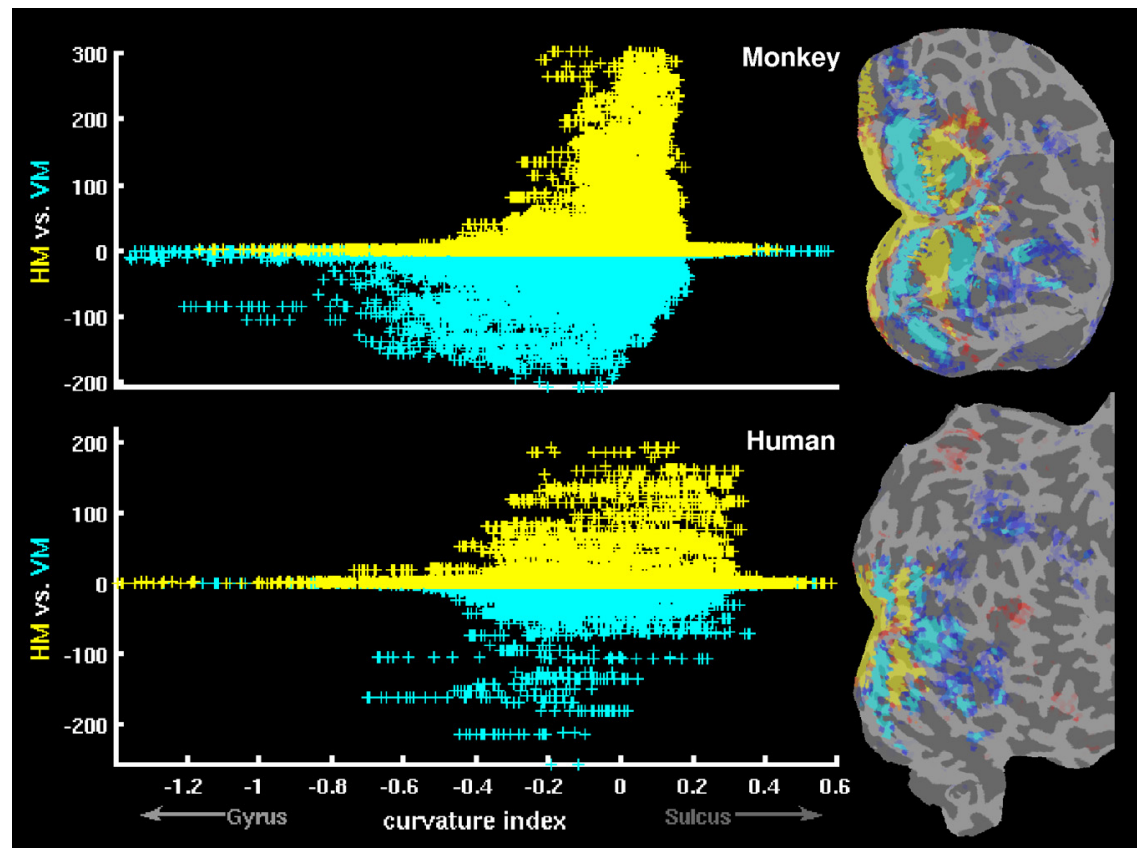

Figure 2. The relationship between meridian maps and cortical folding. The right panels show the relative fMRI activation for horizontal meridian (yellow/red) versus vertical meridian (cyan/blue), displayed on a flattened view of visual cortex from one representative hemisphere in monkeys (top) and humans (bottom). To flatten the cortical surface, the inflated brain was cut (Figurelegend continues.) 
were mostly located in sulci, throughout primate cerebral cortex. On the contrary, an equivalent analysis for eccentricity mapping ("Fov vs Per" contrast) showed no asymmetry in the representation of fovea and periphery on gyri and sulci (supplemental Fig. 1, available at www.jneurosci.org as supplemental material).

Statistically, these differences were quite significant. Figure $3 \mathrm{~A}$ shows the mean curvature (MC) value for HM, VM, Fov, and Per vertices on the cortical surface, as obtained by the weightedaveraging ( $n$, number of vertices; curv, curvature value; $P$, logarithmic $P$ value), as follows:

$$
\mathrm{MC}=\frac{\sum_{i=1}^{n} \operatorname{curv}_{i} P_{i}}{\sum_{i=1}^{n} P_{i}} .
$$

Only vertices with significant $P$ values (i.e., $P>2$ or $P<-2$ ) were included in the MC calculation. The MC values in Figure $3 A$ were based on the averaged data from all monkey and all human hemispheres. This value was positive for HM, indicating a sulcal representation, whereas it was negative for VM, indicating a gyral representation (difference between MC values of HM and VM: $p<0.0001$ in both monkeys and humans, using $t$ test) (see Fig. $3 A$ ). The relationship between meridian mapping and cortical curvature persisted $(p<0.001)$ even when V1 and V2 vertices were excluded from the analysis (Fig. $3 B$ ). This suggests that the "curvature-retinotopy" relationship is a general property throughout retinotopic visual cortex, not limited to the two earliest visual areas. By the same token, this curvature effect in HM and VM nodes was strongest in V1 and progressively weaker in V2 and extrastriate areas (Fig. 3B); this is consistent with the well known decrease in retinotopic order per se, from V1 through progressively higher-tier visual cortical areas. There was an overall gyral bias for both Fov and Per, without statistically significant difference between them ( $p>0.05$ in both monkeys and humans, using $t$ test) (Fig. $3 A$ ). This gyral bias may also reflect technical factors (see supplemental Fig. 1, available at www. jneurosci.org as supplemental material, for details).

\section{Discussion}

These findings support our initial prediction/hypothesis. According to the tension-based model of cortical folding (Van Essen, 1997, 2007), nearby cortical areas with strong topographic connectivity tend to produce a gyrus (an outward fold) at the areal boundary. The border between most retinotopic areas is a vertical meridian representation; this is always the case when an area has a "hemi-field" representation. As a topological constraint for the mirror-symmetric retinotopic areas, the intervening cortex, located between two adjacent vertical meridian representations, is folded in the opposite direction, forming a sulcus (an inward fold) at the region of horizontal meridian representation. This idea assumes that the areal boundaries and the

\footnotetext{
(Figure legend continued.) along the calcarine fissure. Gyral and sulcal cortical folds are shown in light and dark gray, respectively. The scatter plots in the left panels show the relationship between meridian representations and cortical folding in the same hemispheres as shown in the right panels. Each data-point $("+")$ in the scatter plots corresponds to a vertex in the cortical surface, as reconstructed with FreeSurfer. For each vertex, the curvature value ( $x$-axis) and the logarithmic $P$ value for HM versus VM contrast ( $y$-axis) were extracted; then, these values were plotted for all vertices of the entire cortex. The curvature values were based on FreeSurfer's curv file (sulci have positive values, and gyri have negative values). Vertices with positive $P$ values $(\mathrm{HM}>\mathrm{VM})$ are yellow, and vertices with negative $P$ values $(\mathrm{HM}<\mathrm{VM})$ are cyan.
}

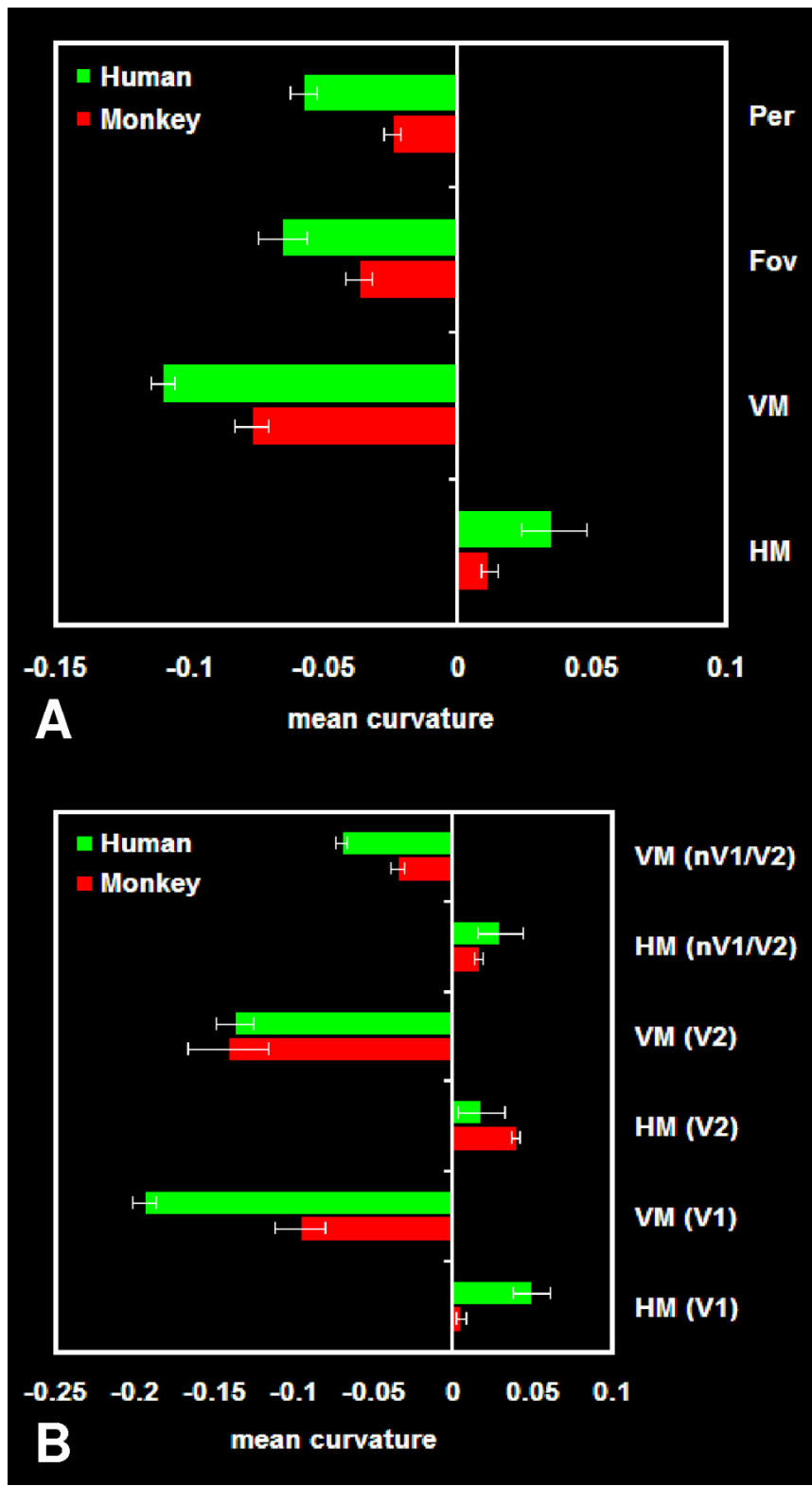

Figure 3. Gyral-sulcal bias for different retinotopic conditions. $\boldsymbol{A}$, The bar plot shows the mean curvature value (see text for formula) for HM, VM, Fov, and Per vertices in the entire visual cortex, averaged across all monkey and all human data. $\boldsymbol{B}$, The bar plot shows the mean curvature value for HM and VM vertices in V1, V2, and "non-V1/V2" (nV1/V2) visual cortex, averaged across all monkey and all human data. The error bars indicate one SEM, as obtained by averaging across hemispheres.

retinotopic maps are defined earlier than gyral/sulcal folds during cortical development.

In addition, the sulcal representation of the horizontal meridian could optimize mapping in the subset areas with "quarterfield" representation (e.g., in V2 and V3). In these areas, a split of the horizontal meridian representation at the V2/V3 border divides each area into dorsal and ventral halves (representing lower and upper visual quadrants, respectively) — thus causing visuotopically neighboring loci across the horizontal meridian to be separated in the two-dimensional cortical map. However, in three-dimensional space, these loci are closer to each other, due to the formation of sulci along the horizontal meridian representation. In the context of the tension-based cortical morphogenesis, the formation of these sulci would require a strong connection between dorsal and ventral cortex 
at/near the horizontal meridian representation of V2 and V3. In fact, a recent study has provided evidence for this (Jeffs et al., 2009).

Our data indicate that these effects occur in both monkeys and humans, suggesting a basic role in the formation of cortical folds. Further studies are needed to establish a causal link between the retinotopic organization of visual cortex and the cortical folding development. Cortical folding may also reflect other factors that are yet to be discovered.

\section{References}

Chklovskii DB, Koulakov AA (2004) Maps in the brain: what can we learn from them? Annu Rev Neurosci 27:369-392.

Jeffs J, Ichida JM, Federer F, Angelucci A (2009) Anatomical evidence for classical and extra-classical receptive field completion across the discontinuous horizontal meridian representation of primate area V2. Cereb Cortex 19:963-981.

Kriegstein A, Noctor S, Martínez-Cerdeño V (2006) Patterns of neural stem and progenitor cell division may underlie evolutionary cortical expansion. Nat Rev Neurosci 7:883-890.

Rajimehr R, Tootell RB (2007) Organization of human visual cortex. In:
The senses: a comprehensive reference, $\mathrm{Vol}$ 1: Vision I (Masland RH, Albright TD, eds), pp 595-614. Oxford: Elsevier.

Rajimehr R, Young JC, Tootell RB (2009) An anterior temporal face patch in human cortex, predicted by macaque maps. Proc Natl Acad Sci U S A 106:1995-2000

Richman DP, Stewart RM, Hutchinson JW, Caviness JVS (1975) Mechanical model of brain convolutional development. Science 189:18-21.

Sereno MI, Tootell RB (2005) From monkeys to humans: what do we now know about brain homologies? Curr Opin Neurobiol 15:135-144.

Vanduffel W, Fize D, Mandeville JB, Nelissen K, Van Hecke P, Rosen BR, Tootell RB, Orban GA (2001) Visual motion processing investigated using contrast agent-enhanced fMRI in awake behaving monkeys. Neuron 32:565-577.

Van Essen DC (1997) A tension-based theory of morphogenesis and compact wiring in the central nervous system. Nature 385:313-318.

Van Essen DC (2007) Cerebral cortical folding patterns in primates: why they vary and what they signify. In: Evolution of nervous systems, Vol 4 (Kaas JH, ed), pp 267-276. London: Elsevier.

Wandell BA, Dumoulin SO, Brewer AA (2007) Visual field maps in human cortex. Neuron 56:366-383.

Welker W (1990) Why does cerebral cortex fissure and fold? A review of determinants of gyri and sulci. In: Cerebral cortex, Vol $8 \mathrm{~b}$ (Jones EG, Peters A, eds), pp. 3-136. New York: Plenum. 